\title{
Instability of Carbolith (lithium carbonate)
}

Reason for posting: A few lots of Carbolith (lithium carbonate) 150-mg capsules were recalled from pharmacies and wholesalers across Canada because of failed stability tests of samples retained beyond 18 months. In November 2004 the manufacturer issued an additional recommendation, endorsed by Health Canada, for physicians to reassess serum lithium concentrations in patients taking Carbolith $150 \mathrm{mg}$. ${ }^{1}$ This precaution should be taken to prevent a possible underdosing and relapse in patients with bipolar disorder, especially in patients whose condition is stabilized with a dose at the lower limit of the therapeutic range.

The drug: Lithium is commonly used and recommended for the treatment and prophylaxis of bipolar disorder, especially the treatment of acute mania and manic episodes. ${ }^{2}$ The mechanism of action of this therapeutic effect is still unclear. Lithium is almost completely eliminated from the body through the kidneys, and how quickly this happens depends on the patient's salt intake, renal function, fluid intake, use of other medications and serum lithium concentration. ${ }^{3}$ Because lithium has a narrow therapeutic range $(0.5-1.2 \mathrm{mmol} / \mathrm{L})$, toxic effects can occur even at therapeutic levels, especially in elderly patients, who usually respond to lower doses $(<1.0 \mathrm{mmol} / \mathrm{L})$. Therefore, the serum concentration of lithium has to be monitored frequently (Box 1). If a patient taking the drug experiences mania or depression, it may be because his or her serum lithium concentration has fallen below $0.5 \mathrm{mmol} / \mathrm{L}$. A worsening of the condition may occur, requiring hospital admission for the patient's own safety or the safety of others. Changing the lithium preparation, however, may result in toxic effects, because another formulation may have a different release time or the patient may not tolerate it well. Tremor of

\section{Box 1: Monitoring patients who are taking lithium ${ }^{4}$}

- Serum lithium levels should usually be measured 3 times weekly and blood studies and urinalysis performed weekly during the initial period of drug administration and as required thereafter. In uncomplicated cases receiving maintenance therapy, serum levels should be monitored at least every 2 months

- Blood samples for serum lithium determination should be drawn immediately before the next dose (12 hours after the last dose)

- Serum creatinine levels should be checked every 2 months, and plasma thyroid hormone and thyroid-stimulating hormone levels every 6-12 months, particularly in female patients

- Periodic monitoring of kidney and cardiovascular function is advisable Therapeutic levels of lithium

- Acute mania: 0.8-1.2 mmol/L

- Maintenance: $0.6-1.0 \mathrm{mmol} / \mathrm{L}$ (elderly patients: $0.4-0.6 \mathrm{mmol} / \mathrm{L}$ )

Levels at which adverse reactions may occur

- 1.5-2.0 mmol/L: mild to moderate reactions

- $>2.0 \mathrm{mmol} / \mathrm{L}$ : moderate to severe reactions

the hands, thirst, frequent urination, drowsiness and blurred vision indicate mild toxic effects; severe toxic effects include confusion, ataxia, vomiting, diarrhea, seizures, coma and even death.

What to do: According to the warning endorsed by Health Canada, physicians should check the serum lithium concentrations of patients who are taking Carbolith $150 \mathrm{mg}$, even if they have no signs of worsening of their condition. If the concentration is below the lower therapeutic limit at which their condition has been stabilized, there is a high risk of relapse. For patients whose condition is stable on maintenance therapy, their serum lithium concentration should be monitored every 2 months on average. ${ }^{3}$

Editorial Fellow, CMAf

\section{References}

1. Health Canada endorsed important safety information on Carbolith $150 \mathrm{mg}$ capsule [Dear Health Care Professional Letter]. Montreal: Valeant Canada Limited; 2004 Nov 4. Available: www.hcsc.gc.ca/hpfb-dgpsa/tpd-dpt/carbolith _hpc_e.html (accessed 2004 Dec 1).

2. Calabrese JR, Kasper S, Johnson G, Tajima O, Vieta E, Yatham LN, et al. International Consensus Group on Bipolar I Depression treatment guidelines. 7 Clin Psychiatry 2004;65(4):571-9.

3. Osborn HH, Malkevich D. Lithium. In: Ford MD, Delaney KA, Ling LJ, Erickson T, editors. Clinical toxicology. Philadelphia: WB Saunders; 2000. p. 532-9.

4. Lithium. In: Compendium of pharmaceuticals and specialties. Ottawa: Canadian Pharmacists Association; 2004. p. 1085-6.

\section{Aleksandra Mi ̌̌ak}

CPS available online

The CPS (Compendium of Pharmaceuticals and Specialties) is now available online. A single-user annual subscription is $\$ 169$ for members of the Canadian Pharmacists Association (CPhA) and $\$ 225$ for nonmembers. Student subscriptions and multi-licence subscriptions for organizations are also available. Contact the CPhA at e-cps@pharmacists.ca for more information. 